\title{
Models of AGN feedback
}

\author{
Françoise Combes \\ Observatoire de Paris \\ 61 Av. de l'Observatoire F-75 014 Paris, France \\ email: francoise. combes@obspm.fr
}

\begin{abstract}
The physical processes responsible of sweeping up the surrounding gas in the host galaxy of an AGN, and able in some circumstances to expel it from the galaxy, are not yet well known. The various mechanisms are briefly reviewed: quasar or radio modes, either momentumconserving outflows, energy-conserving outflows, or intermediate. They are confronted to observations, to know whether they can explain the M-sigma relation, quench the star formation or whether they can also provide some positive feedback and how the black hole accretion history is related to that of star formation.
\end{abstract}

Keywords. galaxies: elliptical and lenticular, cD - galaxies: evolution - galaxies: formation

\section{Introduction}

AGN feedback is invoked to prevent massive galaxies to form in too high abundance, and to understand why the fraction of baryons in galaxies is very low, and decreasing with the galaxy mass (e.g. Behroozi et al. 2013).

It is clear that the growth of massive black holes in the center of galaxies releases enough energy to have a large impact on the galaxy host, if this energy is efficiently coupled to the matter. Assuming the famous relation between black hole and bulge mass (e.g. Gültekin et al. 2009), $\mathrm{M}_{B H}=1-2 \quad 10^{-3} \mathrm{M}_{b u l}$, and the radiative efficiency of accretion onto a black hole of $10 \%, \mathrm{E}_{B H}=0.1 \mathrm{M}_{B H} \mathrm{c}^{2}$, the binding energy of the bulge is $\mathrm{E}_{b u l} \sim \mathrm{M}_{b u l} \sigma^{2}$, with $\sigma$ its velocity dispersion. Typically, the ratio of these two energies is $\mathrm{E}_{B H} / \mathrm{E}_{b u l}>100$. There is therefore no problem in global energy, however, this energy could be radiated away through an elongated cavity perpendicular to the galaxy plane, and the true impact on the galaxy is unknown.

One can distinguish two main modes for AGN feedback (see e.g. the recent review by Fabian, 2012):

- the quasar mode, through radiative processes or winds, when the AGN luminosity is high, close to the Eddington limit. This is the case for young QSOs, at high redshift. The Eddington limit is $\mathrm{L}_{E d d}=4 \pi \mathrm{GM}_{B H} \mathrm{~m}_{p} \mathrm{c} / \sigma_{T}$, with $\sigma_{T}$ being the Thomson scattering cross-section between charged particles and photons. When writing the equilibrium between the gravity and radiative pressure on the infalling ionized gas, combined with the Virial relation, this gives $\mathrm{M}_{B H} \sim \mathrm{f} \sigma_{T} \sigma^{4}$, with $\mathrm{f}$ the gas fraction, which is quite close to the $\mathrm{M}_{B H}-\sigma$ relation. When applying the same considerations at larger radii, this time for neutral gas, submitted to radiation pressure on dust, with the cross-section $\sigma_{d}$, this leads a limitation on the mass than can be accumulated on the bulge and the corresponding masses are in the ratio $\mathrm{M}_{b u l} / \mathrm{M}_{B H}=\sigma_{d} / \sigma_{T} \sim 1000$, which is surprisingly close to the observed ratio.

- the radio mode, or kinetic mode with radio jets, when the AGN luminosity is low, $\mathrm{L}<0.01 \mathrm{~L}_{E d d}$, corresponding typically to low $\mathrm{z}$, massive galaxies, like the local radio ellipticals. The process is then not destructive, and keeps a balance between cooling and heating. This is the mode occuring in cool core clusters, from the AGN of the 
central bright galaxy. It is also associated to low-luminosity AGN in Seyferts. It might be combined with a radiatively inefficient flow ADAF (Advection dominated Accretion Flow).

The best evidence of AGN feedback is found in cool core clusters of galaxies, where the active nucleus in the central galaxy moderates the cooling flow, through its radio jet, creating bubbles in the hot gas. This can have an impact up to radii larger than the central galaxy, at 100kpc scales (McNamara et al. 2009). About 70 percent and possibly 90 percent of clusters have a cool core (Edge et al. 1992). The clusters that are not cooling flows are unrelaxed and most probably recent mergers of sub-clusters.

In the following, we will detail the AGN feedback moderating cooling flows, describe recent results on molecular outflows, driven by starbursts and AGN, and the nature of their mechanisms and energetics. We finish by considerations on the modes of quenching.

\section{AGN feedback moderating gas flow in cool core clusters}

It is frequent in cool core clusters to see the extended impact of the central radio jet, under bubbles and cavities imprinted on the hot X-ray gas, and cold filaments and streaks observed with $\mathrm{H} \alpha$ and $\mathrm{CO}$ emission. In the prototypical Perseus cluster, a large network of cold molecular gas filaments has been observed (Salomé et al. 2006). Recently Canning et al. (2014) have identified a population of very young (a few Myr), compact star clusters, over kiloparsecs scales, related to the network of filaments. These stars could then enrich the stellar halo of the central galaxy NGC 1275.

Observations suggest that the filament network is a consequence of both inflow and outflow. Some of the hot gas cools and fuels the central AGN, which jets entrain the molecular gas in the central galaxy coming from previous cooling episodes. The gas uplifted has been metal enriched in the galaxy before mixing with the cooling gas, and therefore can radiate in $\mathrm{CO}$. The bubbles create inhomogeneities and further cooling. The latter is not only occuring in the center of the cluster, but far away, up to $50 \mathrm{kpc}$, due to the inhomogeneous density and compressions there. The observed velocity of the gas in filaments is much lower than free-fall (Salomé et al. 2008). Numerical simulations have reproduced this process, with buoyant bubbles triggering compression and gas cooling at the surface of cavities, mixing with the cold gas dragged upwards (Revaz et al. 2007).

A large variety of simulations of AGN feedback in clusters or massive elliptical galaxies have been performed, with different degrees of approximaions and sophistication (Brueggen et al. 2007, Cattaneo \& Teyssier 2007, Dubois et al. 2010, Gaspari et al. 2011, 2012). They vary in the cooling rate, or the assumption for accretion (boosted Bondi rate, but cold gas accretion gives better results). None is self-consistent and can predict the accretion rate, which depends on subgrid physics. The radiation pressure mechanism appears insufficient to impact a significant part of the cluster. Mechanical feedback with jets or winds is required. The most recent simulations (Gaspari et al. 2012, see Figure 1), succeed in moderating the cooling, and keep the cool core structure, while previous ones were highly destructive. The efficiency $\epsilon$, ratio between kinetic energy in the jet and accretion energy, can be scaled to the structure, $\epsilon=310^{-4}$ for ellipticals and $510^{-3}$ for clusters.

Are the starbursts related to AGN activity in simulations? Thacker et al. (2014) have performed a comparison between the various models in diagrams of star formation rates (SFR) versus BH accretion rates (BHAR). During a merger, accreted gas fuels both the SFR and BHAR. A BHAR delay is expected since SN feedback is too strong at the beginning (Wild et al. 2010). 

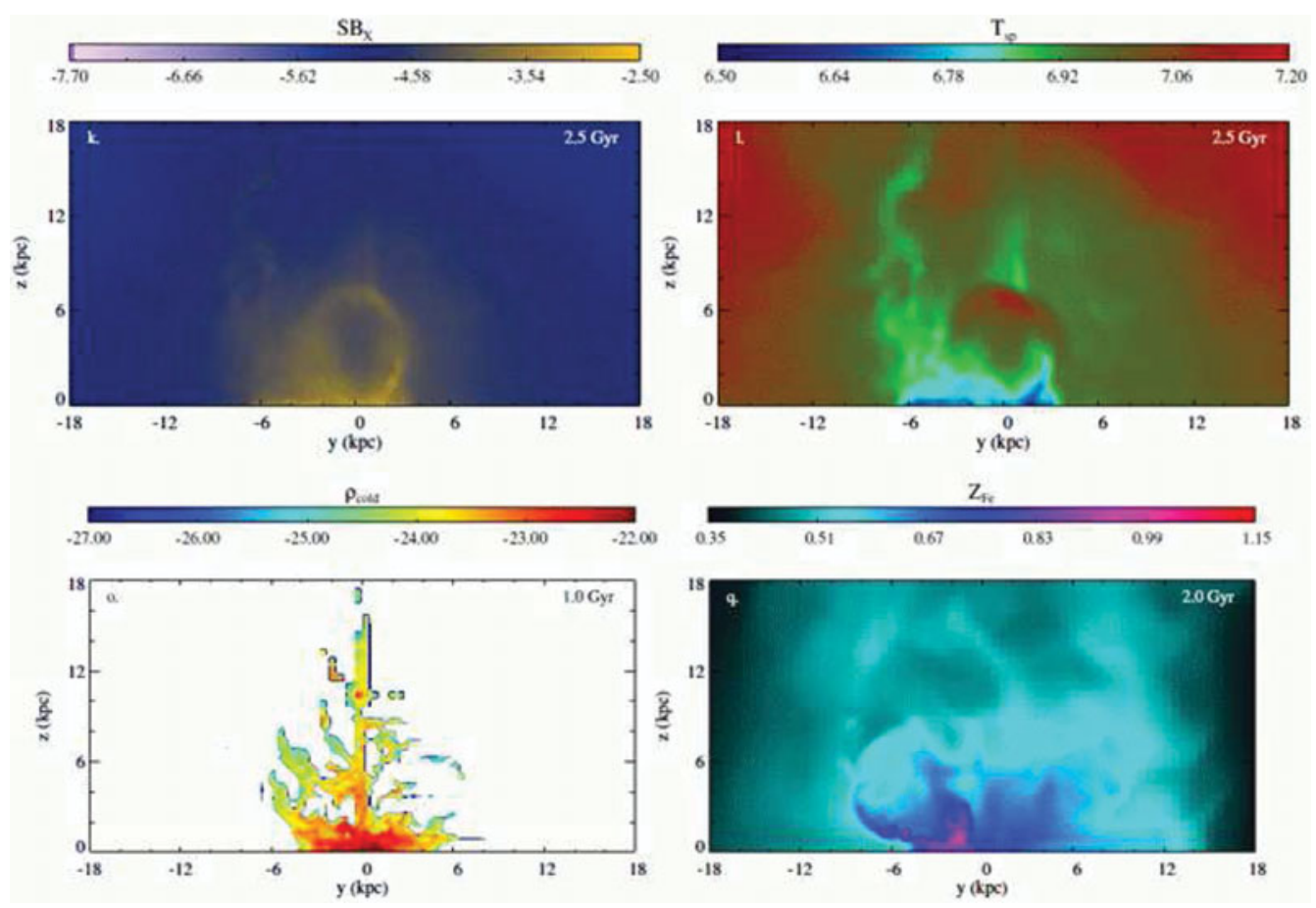

Figure 1. Simulation of AGN feedback in a cool core cluster, with the X-ray surface brightness (top left), the temperature (top right), the cold gas filaments (bottom left) and metallicity (bottom right), from Gaspari et al. (2012).

\section{Molecular Outflows}

In recent years, the discovery of many massive molecular outflows has given support to AGN feedback. In the prototypical Mrk 231 both a nuclear starburst and the AGN contribute to the gas outflow, of $\sim 700 \mathrm{M}_{\odot} / \mathrm{yr}$ (Feruglio et al. 2010). Some outflows have been resolved with a size of a few kpc, significant to impact the galaxy, and provide some quenching. The kinematic power is of the order of $210^{44} \mathrm{erg} / \mathrm{s}$, and the energy of the AGN is required. High density is traced in the outflows through $\mathrm{HCN}, \mathrm{HCO}^{+}$(Aalto et al. 2012). Even more powerful outflows are observed at high redshift (Maiolino et al. 2012).

Cicone et al. (2014) reported interesting relations between outflow rates and AGN properties, for the molecular outflows known. For AGN-hosts, the outflow rate correlates with the AGN power (see Fig 2). The momentum of the outflow is proportional to the photon momentum and is about $20 \mathrm{~L}_{A G N} / \mathrm{c}$. This is interesting to constrain the physical mechanisms of the flow. This boosted momentum can be explained by energy-driven outflows (Zubovas \& King 2012).

The question of the very existence of fast molecular outflows is still unsolved. Outflowing gas is accelerated by a shock, and heated to $10^{6}-10^{7} \mathrm{~K}$. Molecules should be dissociated at such temperatures. Even if cold clumps are carried out in the flow, there should exist some shock signature. One solution proposed is that radiative cooling is quick enough to reform molecules in a large fraction of the outflowing material (Zubovas \& King 2014). With $\mathrm{V} \sim 1000 \mathrm{~km} / \mathrm{s}$, and $\mathrm{dM} / \mathrm{dt} \sim 1000 \mathrm{M}_{\odot} / \mathrm{yr}$, efficient cooling produces multi-phase media, with triggered star formation. 

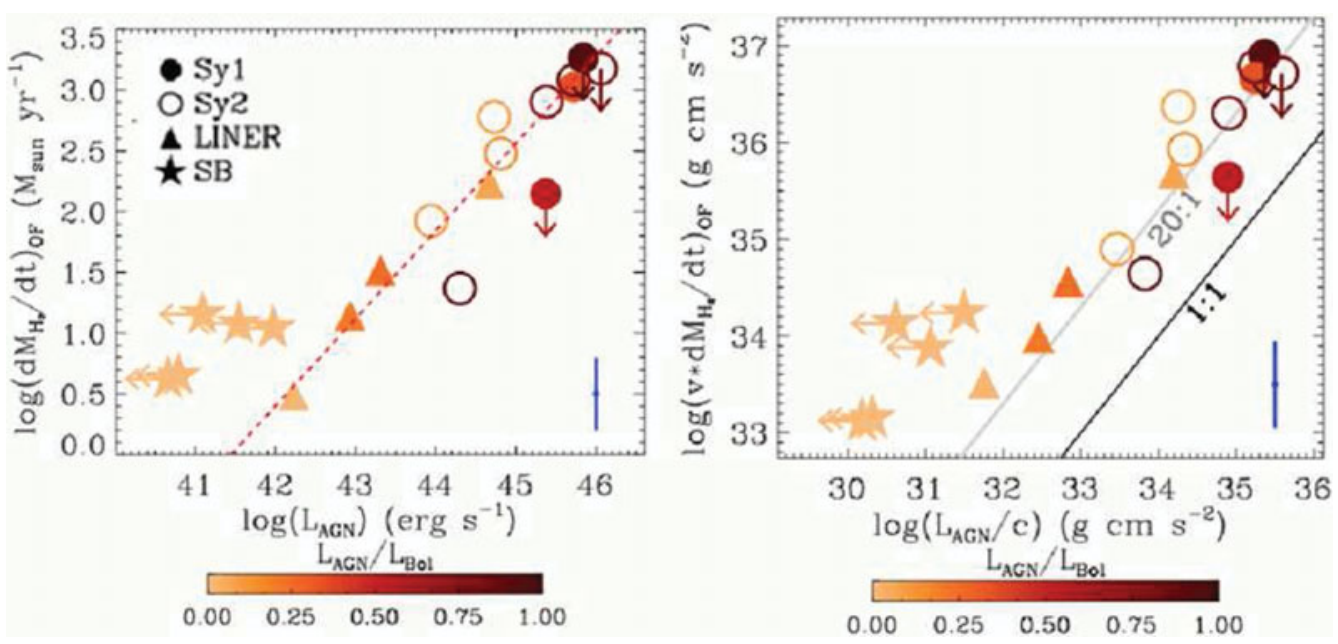

Figure 2. Relation between outflow rate and AGN luminosity (left), and outflow momentum with $\mathrm{L}_{A G N} / \mathrm{c}$, from Cicone et al. (2014). Colors indicate the $\mathrm{L}_{A G N} / \mathrm{L}_{b o l}$ ratio. The various symbols distinguish the AGN types, and the stellar symbols are starbursts.

\section{Mechanisms}

A very schematic view of the shocks generated by an AGN wind is displayed in Fig 3 left. The shocked ISM after the discontinuity region (region c) cools efficiently (freefree emission, metal cooling), and the flow becomes unstable; if the ratio of the radiation pressure to gas pressure is lower than 0.5 , then the gas collapse in clumps, detaching from the hot flow (Krolik et al. 1981). The region (c) becomes multiphase, with Rayleigh-Taylor instabilities. The time-scale for cooling is much lower than $1 \mathrm{Myr}$, and star formation is induced. This may explain the tight correlation between starbursts and AGN. Also it might then be difficult to disentagnle supernovae or AGN outflows. All could have been triggered by the AGN (Zubovas \& King 2014).

\subsection{Energy or momentum conserving outflows}

As shown in Fig 3, if the region (b) of the shocked wind is cooling efficiently, then energy is not conserved, but only the momentum, this is the case of a momentum-conserving outflow.

But for very fast winds $\mathrm{v}_{\text {in }}>10000 \mathrm{~km} / \mathrm{s}$ and up to $50000 \mathrm{~km} / \mathrm{s}$, radiative losses are slow, and almost all the energy can be conserved in this case, called energy-conserving flow (Faucher-Giguère \& Quataert 2012). Then $\mathrm{dM}_{i n} / \mathrm{dt}_{\mathrm{in}}^{2} \sim \mathrm{dM}_{s} / \mathrm{dt}_{s}^{2}$, and there can be a boost in the outflow momentum, as large as $\mathrm{v}_{i n} /\left(2 \mathrm{v}_{s}\right) \sim 50$ ! This explains why the molecular outflows are observed with a momentum flux $>>\mathrm{L}_{A G N} / \mathrm{c}$. It is the push by the hot post-shock gas which boosts the momentum and the velocity $v_{s}$ of the swept-up material. In some cases, even slow winds $\mathrm{v}_{\text {in }} \sim 1000 \mathrm{~km} / \mathrm{s}$ driven by radiation pressure on dust, could be energy-conserving. The phenomenon is analogous to the adiabatic phase, or Sedov-Taylor phase in supernovae remnants. This momentum boost increases the efficiency of supernovae feedback in galaxies.

Within this framework, it was possible to build a realistic model and outflow solution for the typical case of Mrk231, A momentum flux of $15 \mathrm{~L}_{A G N} / \mathrm{c}$ has been obtained (Faucher-Giguère \& Quataert 2012). 

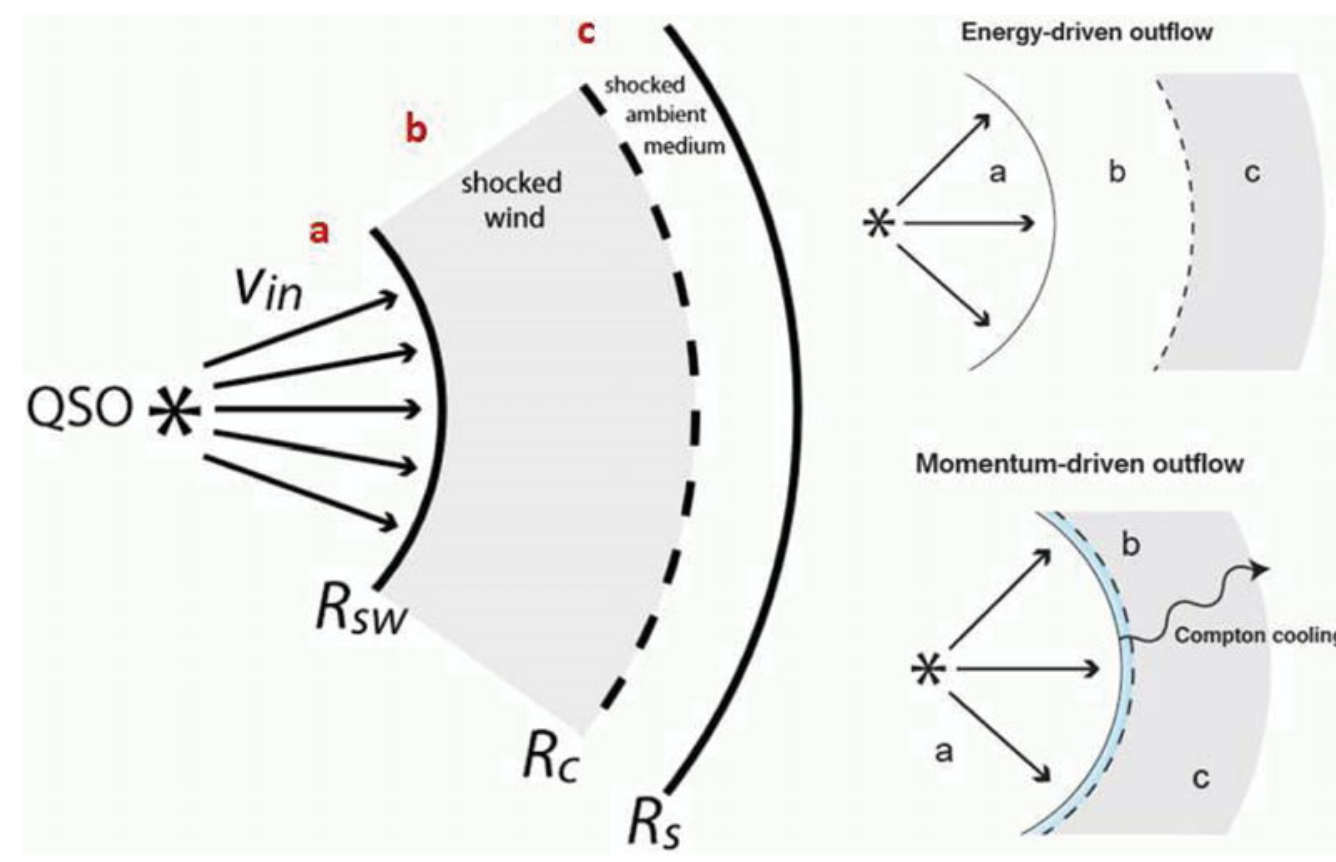

\section{Momentum-driven outflow}

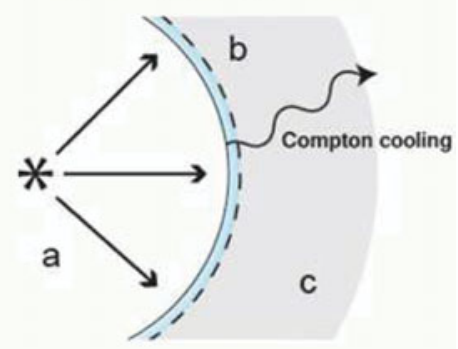

Figure 3. Schematic radial representation of the AGN wind and shock induced in the surrounding ISM. (a) is the wind region; delimited by a reverse shock $\left(\mathrm{R}_{S W}\right)$ (b) is the shocked wind, ending at the discontinuity surface ( $\mathrm{R}_{C}$, dash line), (c) is the shocked ISM, bounded by the forward shock $\left(\mathrm{R}_{S}\right)$, expanding into the unperturbed ISM. The two panels at right indicate the cases of energy-driven outflow (top) where the region (b) is not cooling and energy is conserved there, and momentum-driven outflow (bottom), where the region (b) cools rapidly, loses its pressure support, and becomes thin (from Costa et al. 2014).

\subsection{Winds launch}

Towards the very center, ultra-fast outflows (UFO) or relativistic winds have been observed in UV absorption lines (BAL quasars), or from X-rays coronae (Tombesi et al. 2010, 2014).

These winds can be launched from accretion disks, through several physical mechanisms. Thermal heating (Compton) makes the gas reach the escape velocity. The radiation pressure on electrons (super Eddington regime), or even radiation pressure on dust, or magnetic driving could be the source (Proga 2003, 2005). More realistically, all driving mechanisms may act together.

Simulations of the quasar mode have been performed taking into account the multiphase medium (e.g. Nayakshin 2014). Most of the outflow kinetic energy escapes through the voids. Cold gas is pushed by ram-pressure, but there is more feedback on low-density gas. Both positive and negative feedbacks are observed. The simulations account for the M- $\sigma$ relation.

Simulations of the radio mode have also been performed with a fractal structure of the gas (Wagner \& Bicknell 2011). Relativistic jets produce a very efficient feedback, and impact on the galaxy, in spite of the porosity of the ISM.

\subsection{Positive AGN feedback}

Many simulations reveal signs of positive feedback (e.g. Silk 2005, Dubois et al. 2013). The phenomenon is more difficult to observe, but some systems do show evidence of jetinduces star formation, like the Minkowki object, Centaurus A, or for instance the young, 


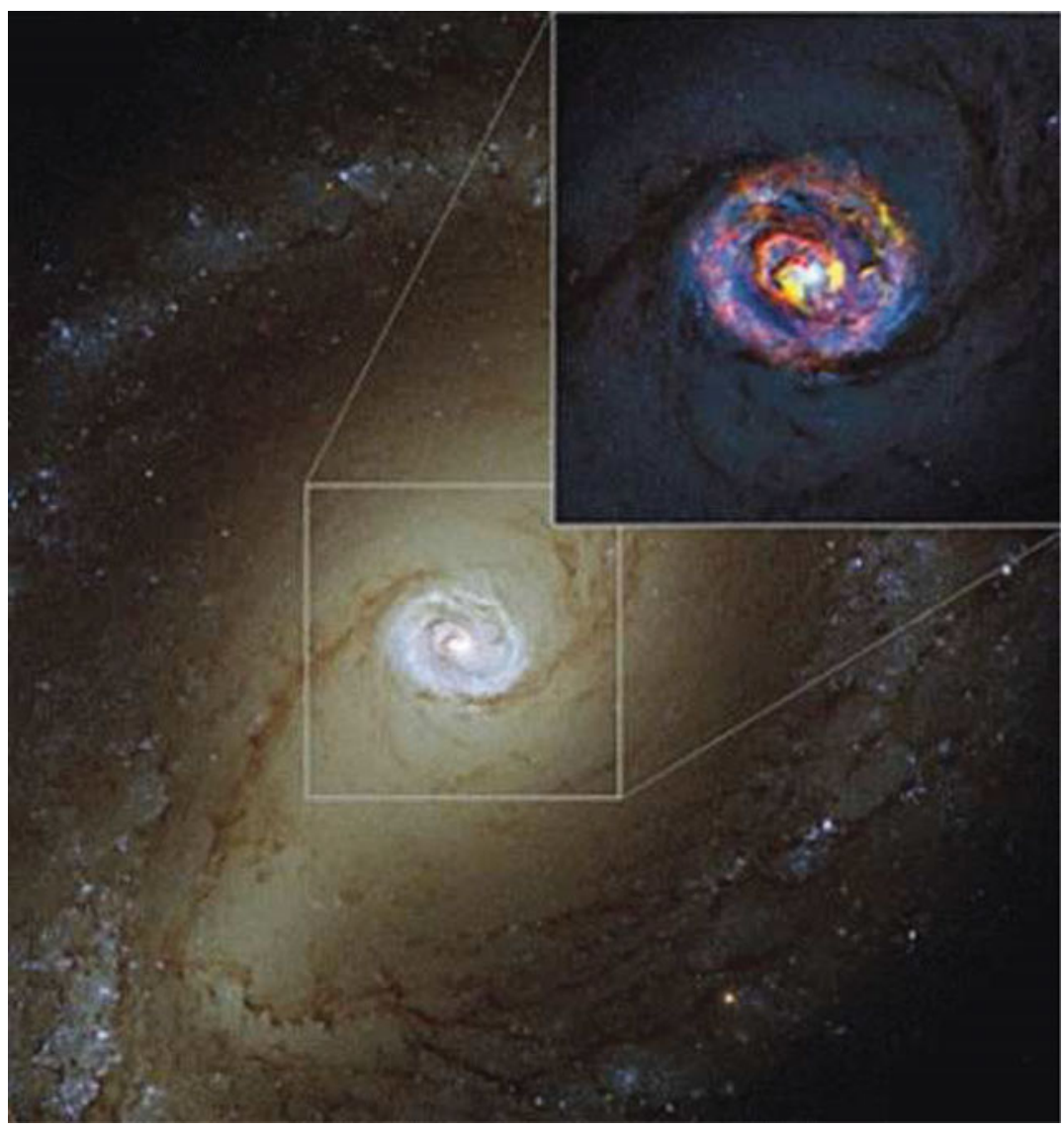

Figure 4. The NGC 1433 barred spiral is a Seyfert 2 of low-luminosity. The CO(3-2) ALMA map of the nuclear disk shown here superposed on the HST image has revealed an outflow on the minor axis, the smallest molecular flow detected up to now (Combes et al. 2013).

restarted radio loud AGN 4C12.50. The outflow is located $100 \mathrm{pc}$ from the nucleus where the radio jet interacts with the ISM (Morganti et al. 2013, Dasyra \& Combes 2012).

\subsection{Feedback in low-luminosity $A G N$}

Feedback can also play a role in low-luminosity AGN, in moderating star formation. The modest impact here is compensated by the large numbers of such objects. Recently the ALMA observations at $25 \mathrm{pc}$ resolution of the $\mathrm{CO}(3-2)$ line in the barred spiral NGC 1433 has revealed a molecular ouflow on the minor axis (Combes et al. 2013, see Fig 4.). About $7 \%$ of the molecular mass detected belongs to the outflow, with a velocity $100 \mathrm{~km} / \mathrm{s}$, uncertain because of ill-known inclination. This is the smallest flow detected up to now. The kinetic luminosity is $\sim 2.310^{40} \mathrm{erg} / \mathrm{s}$, and the flow momentum is $>10$ $\mathrm{L}_{A G N} / \mathrm{c}$.

The molecular gas is still fueling the AGN, as shown by the computed gravity torques of the bar (Smajic et al. 2014).

\section{Modes of quenching}

There are two main modes to consider, a rapid one, with time-scales of $100 \mathrm{Myr}$, in galaxy mergers, with feedback from supernovae and from AGN, and a slow one, with 
2-4 Gyr time-scales, either through morphological quenching after bulge formation, or through strangulation, where the replenishment of the disk in gas is stopped (Schawinski et al. 2014). Mild feedback could also delay star formation, in a secular way. It is however observed that the co-evolution of galaxies and black holes is no longer tight for low-mass galaxies, governed by secular evolution (e.g. Kormendy \& Ho 2013).

It is not sure whether a feedback loop is required to explain the M- $\sigma$ relation. Simulations of torque limited growth of the central $\mathrm{BH}$ have retrieved the relation, without any feedback (Angles-Alcazar et al. 2013).

The galaxy and BH grow mostly through gas accretion, sometimes mergers (but they are not essential). The numerical evolution has been computed with different M- $\sigma$ relations for seeds. However, the effects of initial conditions are quickly erased. The growth of the $\mathrm{BH}, \mathrm{dM}_{B H} / \mathrm{dt} \sim \mathrm{SFR}$ with scatter, and no feedback loop is required.

AGB feedback in mergers have been invoked for a long time for theoretical reasons, and simulated with the usual sub-grid physics (e.g. Springel et al. 2005, Hopkins et al. 2006). However, the amount of feedback, and how efficiently it couples with the galaxy disks is unknown. Recently, Gabor \& Bournaud (2014) claim that the AGN quenching effect on star formation is negligible, although a significant amount of hot gas is expelled in the intergalactic medium.

Several modes have been simulated, with more sophisticated details: quasar mode, when $\mathrm{dM}_{B H} / \mathrm{dt}>0.01$ Eddington rate, and the energy is released spherically; radio-jets otherwise, with a velocity $\mathrm{V}=10^{4} \mathrm{~km} / \mathrm{s}$ imposed in a cylinder perpendicular to the disk.

The efficiency to form stars can be reduced by a factor 7 , and simulations do show a decrease of the baryon concentration (Dubois et al. 2013).

Costa et al. (2014) have reproduced the two cases energy and momentum driven winds, and find the energy-driven mode much more efficient, with outflow momenta $>10 \mathrm{~L}_{E d d} / \mathrm{c}$. The entrained cold gas has masses $>10^{9} \mathrm{M}_{\odot}$ if the after-shock gas cools with metals.

\section{Conclusions}

AGN feedback is very efficient in cool core clusters to moderate the cooling. The mechanism here is mechanical with radio jets and cold gas accretion.

Molecular outflows are now observed frequently around AGN, with velocities $\mathrm{v}=200$ $1200 \mathrm{~km} / \mathrm{s}$, outflowing masses $10^{7}-10^{9} \mathrm{M}_{\odot}$, and load factors $>3$.

The mechanisms range from quasar modes (winds) with powerful AGN, to radio modes (jets), for more massive galaxies with lower Eddington ratios (and lower redshift). The fueling could be either cosmic mass accretion or mergers.

Molecular outflows in AGN-hosts reveal boosted momentum flux of $\sim 20 \mathrm{~L}_{A G N} / \mathrm{c}$ This can only be obtained through energy-conserving flows.

\section{Acknowledgements}

Thanks for the organisers, and in particular the chair Bodo Ziegler for such an exciting and well-organised conference. I acknowledge the European Research Council for the Advanced Grant Program Number 267399-Momentum.

\section{References}

Aalto, S., Garcia-Burillo, S., Muller, S. et al., 2012, A\&A 537, A44

Angles-Alcazar, D., Ozel, F., \& Davé, R., 2013, ApJ 770, 5

Behroozi, P. S., Wechsler, R. H., \& Conroy, C., 2013, ApJ 770, 57 
Brueggen, M., Heinz, S., Roediger, E., et al., 2007, MNRAS 380, L67

Canning, R. E. A., Ryon, J. E., Gallagher, J. S., et al., 2014, MNRAS in press, arXiv1406.4800 Cattaneo, A. \& Teyssier, R., 2007, MNRAS 376, 1547

Cicone, C., Maiolino, R., Sturm, E., et al., 2014, A\& $A$ 562, A21

Combes, F., Garcia-Burillo, S., Casasola, V., et al., 2013, A $\mho A$ A58, A124

Costa, T., Sijacki, D., \& Haehnelt, M. G., 2014, MNRAS in press arXiv1406.2691

Dasyra, K. \& Combes F., 2012, A\& A 541, L7

Dubois, Y., Devriendt, J., Slyz, A., \& Teyssier, R., 2010, MNRAS 409, 985

Dubois, Y., Pichon, C., Devriendt, J., et al., 2013, MNRAS 428, 2885

Edge, A. C., Stewart, G. C., \& Fabian, A. C., 1992, MNRAS 258, 177

Fabian, A. C., 2012, ARAA 50, 455

Faucher-Giguère, C-A. \& Quataert, E., 2012, MNRAS 425, 605

Feruglio, C., Maiolino, R., Piconcelli, E., et al., 2010, A\&A 518, L155

Gabor, J. \& Bournaud, F., 2014, MNRAS 441, 1615

Gaspari, M., Melioli, C., Brighenti, F., \& D'Ercole, A., 2011, MNRAS 411, 349

Gaspari, M., Brighenti, F., \& Temi, P., 2012, MNRAS 424, 190

Gültekin, K., Richstone, D. O., Gebhardt, K., et al., 2009, ApJ 698, 198

Hopkins, P., Hernquist, L., Cox, T. J., et al., 2006, ApJS 163, 1

Kormendy, J. \& Ho, L. C., 2013, ARAA 51, 511

Krolik, J. H., McKee, C. F., \& Tarter, C. B., 1981, ApJ 249, 422

Maiolino, R., Gallerani, S., Neri, R., et al., 2012, MNRAS 425, L66

McNamara, B. R., Kazemzadeh, F., Rafferty, D. A., et al., 2009, ApJ 698, 594

Morganti, R., Fogasy, J., Paragi, Z., et al., 2013, Science 341, 1082

Nayakshin, S., 2014, MNRAS 437, 2404

Proga, D. 2003, ApJ 585, 406

Proga, D. 2005, ApJ 630, L9

Revaz, Y., Combes, F., \& Salomé P., 2007, A\&A 477, L33

Salomé, P., Combes, F., Edge, A., et al., 2006, A\&A 454, 437

Salomé, P., Combes, F., Revaz, Y., et al., 2008, A\&A 484, 317

Schawinski, K., Urry, C. M., Simmons, B. D., et al., 2014, MNRAS 440, 889

Silk, J., 2005, MNRAS 364, 1337

Smajic, S., Moser, L., Eckart, A., et al., 2014, A\&A in press, arXiv1404.6562

Springel, V., Di Matteo, T., \& Hernquist, L., 2005, MNRAS 361, 776

Thacker, R. J., MacMackin, C., Wurster, J., \& Hobbs, A., 2014, MNRAS in press, arXiv1407.0685

Tombesi, F., Cappi, M., Reeves, J. N., et al., 2010, A\&A 521, A57

Tombesi, F., Tazaki, F., Mushotzky, R. F., et al., 2014, MNRAS in press arXiv1406.7252

Wagner, A. Y. \& Bicknell, G. V., 2011, ApJ 728, 29

Wild, V., Heckman, T., \& Charlot, S., 2010, MNRAS 405, 933

Zubovas, K. \& King, A., 2012, ApJ 745, L34

Zubovas, K. \& King A., 2014, MNRAS 439, 400 